\title{
Development and Regulation of Medical Internet
}

\author{
$\mathrm{Na} \mathrm{Wang}^{1, \mathrm{a}}$ and corresponding author: Jinguo Wang ${ }^{2, \mathrm{~b},{ }^{*}}$ \\ ${ }^{1}$ Department of Anesthesiology, The First Hospital of Jilin University, Changchun, China \\ ${ }^{2}$ Department of Urology, The First Hospital of Jilin University, Changchun, China \\ awangna080613@163.com, bwangjinguolily@163.com
}

Keywords: Development, Regulation, Medicine, Internet.

Abstract. The development of medical Internet is fast, so the related laws and regulations fail to keep up with the pace of its development. If a doctor practices online, there is no clear regulation and rules. At the same time, domestic clients haven't the habit to pay for the medical service from the Internet. The Internet groups also use mobile phone, but who are willing to pay for the software of mobile device is relatively few. It is current national condition in our country to use software without fees in the form of operation. In some extents, the Internet medical profit model is not perfect.

\section{Introduction}

Medical Internet is a significant characteristic of Internet in medical service industry. It embodies the improvement of the health care industry from the Internet. Through the transparency of the Internet and convenience to integrate the existing medical resources, the medical industry can solve the existing difficulties. [1]

In popular terms, medical Internet care means to meet the needs of public medical services on the Internet platform, disseminate health knowledge and provide health consultation and management services to the public. [2] The rise of medical Internet treatment is not only the inevitable trend of the development of the society, but also the value of its intrinsic function.

Chinese Internet medical industry has a large market space and rich in all kinds of data, good foundation of medical and health information, the Internet (especially the mobile Internet penetration rate is high, the abundant social capital, the favorable factors such as relative perfect capital market, but also facing applications how to deepen the development, imperfect legal policy regulation, user privacy cannot be guaranteed, medical disputes have occurred and the difficulty of information share between medical institutions, a series of problems. In this context, once the bottleneck has reached a breakthrough, China's Internet medical industry will have a great development. [3]

\section{Industrial Transformation of the Internet}

The Origin of Internet Medical Care. As early as the last century, It has developed a prototype of using the Internet to provide medical services in China, and has undergone the transformation of information collection and management from health knowledge promotion and consultation to personal health. [4] However, due to the early information technology and its surrounding industry information is in the initial stage, this stage of the Internet medical service mainly through information data collection and assessment

At present, the source of information is narrow and the accuracy is limited, which leads to the insufficient effectiveness of the service. In recent years, with the development of Internet and information technology, appeared such as wearable device extremely significance of new products, greatly expand the source of personal health information, the direct source of information to ensure the authenticity and accuracy of information, in order to achieve targeted medical services. [5] The Internet's blustery also drove the development of peripheral industries, such as mobile Internet, big data, and the Internet of things emerging technologies such as gradually maturing, the information collection analysis ability is greatly increased, offline resources platform integration than in the past have significantly improved their consciousness and ability, which laid a technical foundation for the rise of Internet medical treatment. [6] 
The Information Development of Hospital. Just need to residents' health demand, is the needs of other irreplaceable, especially improve the income level of residents in our country, under the condition of residents' health demand increasingly strong. The growing demand for the health not only embodies in total, more important is the quality of the residents demands for health promotion, namely the residents not only need better drug curative effect, more high-end medical diagnostic equipment, need more high quality service facilities and medical management services. At present, the key factor restricting the health demand of Chinese residents is unreasonable allocation of medical resources. [7]

\section{Application Modes}

Internet medical care aims to achieve the in-depth integration of medical resources and patient needs through the Internet, strengthen the connectivity of the whole industry chain, optimize the process, enhance the user experience, and achieve full process coverage. [8]

But because of medical health service chain is longer, and the electronic prescription outflow, net selling prescription drugs, health care payments, the restriction of various factors such as information sharing, at present China's Internet medical each link are relatively independent, poor service and data sharing process, which behaves particularly outstandingly in the courtyard outside disconnect. [9] In this situation, it is difficult for Internet medical treatment to truly change the entire medical service process, and its core value cannot be truly reflected.

Identification Requirements for Information. Unified management of the identity data of employees, the management of basic dictionaries and user accounts, is the basic work of unified login authentication for mobile medical users in the hospital. Unified management in patients with wrist strap identification information, combined with the basic information of the patients EMPI is used, the more effective medical information system linked together, guarantee for the same patient, the integrity of the distribution in different system of personal information collection and accuracy. [10]

Using one or two dimensional barcode, RFID radio-frequency identification technology, identifies the information of drugs, biochemical specimens, equipment, medical staff and patient status. Barcode scanning can not only confirm the correspondence of information quickly but also effectively eliminate the errors of human judgment. [11]

Management Requirements of Mobile Healthcare Access and Update. The patient's personal health profile information is scattered throughout the hospital, community application system, and even some health follow-up information is generated in the patient's home medical device. With the aid of information platform, integrated health life cycle all diagnostic information, including all kinds of test results, radiation check images, prescription, follow-up, and health monitoring data information, such as clinical medical service personnel can timely understanding of service object complete medical activity, is helpful to improve the level of medical treatment, to reduce the medical risk. [12]

Service Content. Geared to the needs of different subjects, different link, from the periphery to the core permeability Internet medical treatment for patients, doctors, hospitals, pharmaceutical companies, insurance companies and administrative supervision department six main body, and the health management, pre-hospital consulting, leading examining registered waiting, diagnosis, pay, prescription drugs, treatment, hospitalization and rehabilitation of different links such as after school.

Currently geared to the needs of different subjects, different links all have different applications, such as health management and rehabilitation, slow disease management, online profile, auxiliary treatment (registered, leading examining, triage), hospital, medicine, electricity, Internet, doctor tools, support platform, etc. These applications have improved the old health care services in varying degrees. [13]

Due to the characteristics of medical services in China determines the current domestic Internet equal medical applications to medical resources and improve the efficiency of the service as a starting point, therefore, make an appointment, medicine, electricity and other auxiliary medical application in improving efficiency, convenient side effect is obvious. [14] 
Wearable devices, cloud storage and service, and big data management and to the health of the residents of the combination of rehabilitation provides a new means and support, so residents can be more easily for health management and rehabilitation. Doctors' tools to help doctors get rid of onerous medical services. The analysis and decision making applications of the insurance and administrative departments are also playing their roles in online inquiry, PBM and service full process information collection.

Regional Collaborative Medicine. The regional cooperative medical treatment, it is to point to in a certain area range, integration of various medical resources, the use of information technology, medical technology, mutual collaboration and resource sharing between medical institutions, and to maximize the medical resource utilization.

In recent years, through the construction of regional health information platform, it realizes the integration of medical resources and business system connectivity between medical institutions, reduces the repeated examination and improves disease management and care.

At the same time, the abundant medical resources of large comprehensive hospitals are radiated to the basic medical institutions, which can help optimize the allocation of medical resources and promote the classification and treatment. [15]

The study found that the medical Internet can provide abundant technical support, because convenient service should actively use various new technologies, promote and benefit a national health information services and the wisdom of the medical service.

\section{Conclusions}

To sum up, the Internet got rapid development in medical health care industry in our country, has initially formed a variety of service mode, to improve the quality of online medical service and promote medical staff communication study has important significance. In order to promote the healthy development of the medical industry, the Internet must further strengthen the supervision of the industry, gradually exploring mature profit model in practice, create a good industry operation environment, guarantee of medical institutions, the third party institution and the lawful rights and interests of the medical staff and patients.

\section{References}

[1] Liangzhao Zeng, Boualem Benatallah, Anne H.H. Ngu, Marlon Dumas, Jayant Kalagnanam, Henry Chang. QoS-Aware Middleware for Web Services Composition. IEEE Transactions on Software Engineering. 2004

[2] Alexandros Labrinidis, H. V. Jagadish. Challenges and opportunities with big data. Proceedings of the VLDB Endowment. 2012

[3] George Lee, Jimmy Lin, Chuang Liu, Andrew Lorek, Dmitriy Ryaboy. The unified logging infrastructure for data analytics at Twitter. Proceedings of the VLDB Endowment. 2012

[4] Peter Mell, Tim Grance. The NIST Definition of Cloud Computing. Communications of the ACM. 2010

[5] Sara Cohen, Carmel Domshlak, Naama Zwerdling. On ranking techniques for desktop search[J]. ACM Transactions on Information Systems (TOIS). 2008 (2)

[6] A.Pavlo, E.Paulson, A.Rasin. A Comparison of approaches to large-scale data analysis. Proc of the 2009 ACM SIGMOD Int Conf on Management of Data. 2009

[7] Lv Q, Cao P, Cohen E. Search and replication in unstructured peer-to-peer networks. Proceedings of the 16th ACM International Conference on Supercomputing (ICS`02). 2002 
[8] Sylvia Ratnasamy, Paul Francis, Mark Handley. A scalable content-addressable network. Proceedings of the 2001 Conference on Applications, Technologies, Architectures, and Protocols for Computer Communications (SIGCOMM 2001). 2001

[9] Marco Crasso, Alejandro Zunino, Marcelo Campo. Easy web service discovery: A query-by-example approach[J]. Science of Computer Programming. 2008 (2)

[10] Joyce E Hadad, Maude Manouvrier, Marta Rukoz. TQoS: Transactional and QoS-Aware Selection Algorithm for Automatic Web Service Composition. IEEE Transactions on Services Computing. 2010

[11] Ripeanu M. Peer-to-peer architecture case study: Gnutella network. Proceedings of the First International Conference on Peer-to-Peer Computing(P2P'01). 2001

[12] Michael J. Carey, Nicola Onose, Michalis Petropoulos. Data services[J]. Communications of the ACM. 2012 (6)

[13] Wei Li, Bo Lang. A tetrahedral data model for unstructured data management[J]. Science China Information Sciences. 2010 (8)

[14] Jing H, Haihong E, Guan L, Jian D. Survey on NoSQL database. Pervasive Computing and Application (ICPCA),2011 6th IEEE International Conference. 2011

[15] Kyriakos Kritikos, Dimitris Plexousakis. Mixed-Integer Programming for QoS-Based Web Service Matchmaking. IEEE Transactions on Services Computing. 2009 